\title{
Deep sequencing and expression of microRNAs from early honeybee (Apis mellifera) embryos reveals a role in regulating early embryonic patterning
}

\author{
Lisa Zondag ${ }^{1,2}$, Peter K Dearden ${ }^{1}$ and Megan J Wilson ${ }^{1,2^{*}}$
}

\begin{abstract}
Background: Recent evidence supports the proposal that the observed diversity of animal body plans has been produced through alterations to the complexity of the regulatory genome rather than increases in the protein-coding content of a genome. One significant form of gene regulation is the contribution made by the non-coding content of the genome. Non-coding RNAs play roles in embryonic development of animals and these functions might be expected to evolve rapidly. Using next-generation sequencing and in situ hybridization, we have examined the miRNA content of early honeybee embryos.

Results: Through small RNA sequencing we found that 28\% of known miRNAs are expressed in the early embryo. We also identified developmentally expressed microRNAs that are unique to the Apoidea clade. Examination of expression patterns implied these miRNAs have roles in patterning the anterior-posterior and dorso-ventral axes as well as the extraembryonic membranes. Knockdown of Dicer, a key component of miRNA processing, confirmed that miRNAs are likely to have a role in patterning these tissues.

Conclusions: Examination of the expression patterns of novel miRNAs, some unique to the Apis group, indicated that they are likely to play a role in early honeybee development. Known miRNAs that are deeply conserved in animal phyla display differences in expression pattern between honeybee and Drosophila, particularly at early stages of development. This may indicate miRNAs play a rapidly evolving role in regulating developmental pathways, most likely through changes to the way their expression is regulated.
\end{abstract}

\section{Background}

A major component of the transcriptome of animals consists of non-protein coding RNAs [1-3]. Micro-RNAs (miRNAs) are a subset of small non-coding RNAs that are 18-24 nucleotides long and have a key role in regulating gene expression in eukaryotes. They are produced from a primary full-length transcript (pri-miRNA), which is cleaved to form hairpin structures around 70 nucleotides in length. These are called precursor miRNAs (pre-miRNAs) and are exported to the cytoplasm

\footnotetext{
* Correspondence: meganj.wilson@otago.ac.nz

'Laboratory for Evolution and Development, Genetics Otago and the National Research Centre for Growth and Development, Department of Biochemistry, University of Otago, P.O. Box 56, Dunedin 9054, New Zealand ${ }^{2}$ Developmental Biology Laboratory, Department of Anatomy, University of Otago, P.O. Box 56, Dunedin 9054, New Zealand
}

to be processed further to functional mature miRNAs by the ribonuclease Dicer $[4,5]$. Once assembled into the RNA-induced silencing complex (RISC), the miRNA acts on its target by binding to complementary sequences present in the $3^{\prime}$ untranslated regions (UTR) of the target mRNA [6]. This results in either translational repression or mRNA cleavage, thus providing another level of gene regulation [7].

There is accruing evidence to suggest that miRNAs play a role in regulating multiple developmental pathways, including fundamental developmental processes of animal development such as axis formation and organ morphogeneis. Many miRNAs are expressed in developmentally restricted patterns [8,9], for example, examination of miRNA expression in Drosophila during embryogenesis found that many are expressed in

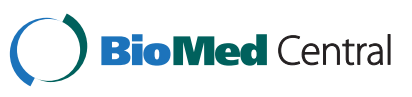


Table 1 Summary of small RNA sequencing results

\begin{tabular}{lll}
\hline & Sample 1 & Sample 2 \\
\hline Total number of reads & 20120856 & 21480569 \\
\hline Clean reads & 19563073 & 20873296 \\
\hline Number of reads mapped back to genome & 13200933 & 14892414 \\
\hline Number of reads map to annotated pre-miRNA & 21450 & 18469 \\
\hline
\end{tabular}

restricted patterns along the anterior-posterior and dorso-ventral axes and in specific tissues. Their expression is developmentally regulated, often by their own promoter and regulatory elements, similar to developmental protein coding factors [10]. Loss of miRNA function often results in defective development and patterning [11-14], indicating an essential role in animal development. MiRNAs are also proposed to provide developmental stability particularly under times of environment stress, acting to buffer developmental pathways [15].

Many miRNA families are ancient and can be traced to more basal animals. Expression analysis and functional studies indicate that their roles are often conserved [8]. As single miRNAs can bind to many different mRNAs to regulate their expression, they can potentially impact on several regulatory pathways [16]. Thus any changes to the way miRNAs are expressed are likely to impact multiple developmental processes. Many miRNAs have been found to be specific to particular phylogenetic groups, some found only in particular lineages. Over 40 miRNA families arose early in the lineage leading to the vertebrates, and it has been suggested that these contributed to the evolution of vertebrate complexity [17].

Here we have profiled the miRNAs expressed during early embryogenesis in the honeybee (Apis mellifera) to determine if they are likely to play a significant role in honeybee embryogenesis. The expression and function of miRNAs in insect development has to date only been investigated in Tribolium and Drosophila $[8,18]$. Like Drosophila, Apis development begins with a syncytial blastoderm stage prior to cellularisation, where much of the body patterning information is established [19]. Both Apis and Drosophila are considered long germ band insects where segmentation occurs across the whole body [20]. However there are some significant differences, notably in patterning of the extraembryonic membranes. In Drosophila the extraembryonic membranes are patterned as one tissue, the amino-serosa, a process regulated by the transcription factor zen [21]. In honeybee, these membranes are patterned separately, although both tissues still require zen [22]. There are also significant differences in the nature of the regulatory networks required to pattern the anterior-posterior axis [22-24]. The honeybee genome has been sequenced and 168
Table 2 Profile of known miRNAs in present in 24-30 hour embryos

\begin{tabular}{|c|c|c|}
\hline miRNA & Sample 1 & Sample 2 \\
\hline ame-miR-100 & 101 & 73 \\
\hline ame-miR-1 & 94 & 5 \\
\hline ame-miR-71 & 269 & 88 \\
\hline ame-miR-3759 & 5 & 107 \\
\hline ame-miR-184 & 5294 & 4431 \\
\hline ame-miR-927 & 21 & 22 \\
\hline ame-miR-275 & 22 & 15 \\
\hline ame-miR-7 & 158 & 73 \\
\hline ame-miR-279 & 23 & 8 \\
\hline ame-miR-8 & 9 & 8 \\
\hline ame-miR-92b & 103 & 149 \\
\hline ame-miR-283 & 8 & 16 \\
\hline ame-miR-3756 & 362 & 264 \\
\hline ame-miR-2 & 167 & 261 \\
\hline ame-miR-263b & 6 & 16 \\
\hline ame-miR-3785 & 13 & 14 \\
\hline ame-miR-11 & 15 & 26 \\
\hline ame-miR-3747a & 606 & 143 \\
\hline ame-miR-279c & 544 & 310 \\
\hline ame-miR-315 & 4 & 13 \\
\hline ame-miR-13a & 10 & 3 \\
\hline ame-miR-12 & 8 & 20 \\
\hline ame-miR-2944 & 2452 & 1177 \\
\hline ame-miR-263 & 39 & 141 \\
\hline ame-miR-375 & 6 & 6 \\
\hline ame-miR-279b & 335 & 318 \\
\hline ame-miR-34 & 9 & 6 \\
\hline ame-miR-750 & 8 & 7 \\
\hline ame-miR-989 & 8 & 4 \\
\hline ame-miR-9c & 89 & 69 \\
\hline ame-miR-3477 & 211 & 199 \\
\hline ame-miR-3715 & 15 & 35 \\
\hline ame-miR-10 & 3923 & 3561 \\
\hline ame-miR-125 & 49 & 87 \\
\hline ame-miR-3747b & 63 & 23 \\
\hline ame-miR-996 & 17 & $\overline{3}$ \\
\hline ame-miR-9a & 2214 & 1977 \\
\hline ame-miR-92a & 175 & 214 \\
\hline ame-let-7 & 135 & 185 \\
\hline ame-miR-3786 & 31 & 32 \\
\hline ame-miR-252 & 14 & 10 \\
\hline ame-bantam & 13 & 6 \\
\hline ame-miR-317 & 360 & 354 \\
\hline ame-miR-306 & 608 & 619 \\
\hline ame-miR-3791 & 592 & 707 \\
\hline
\end{tabular}




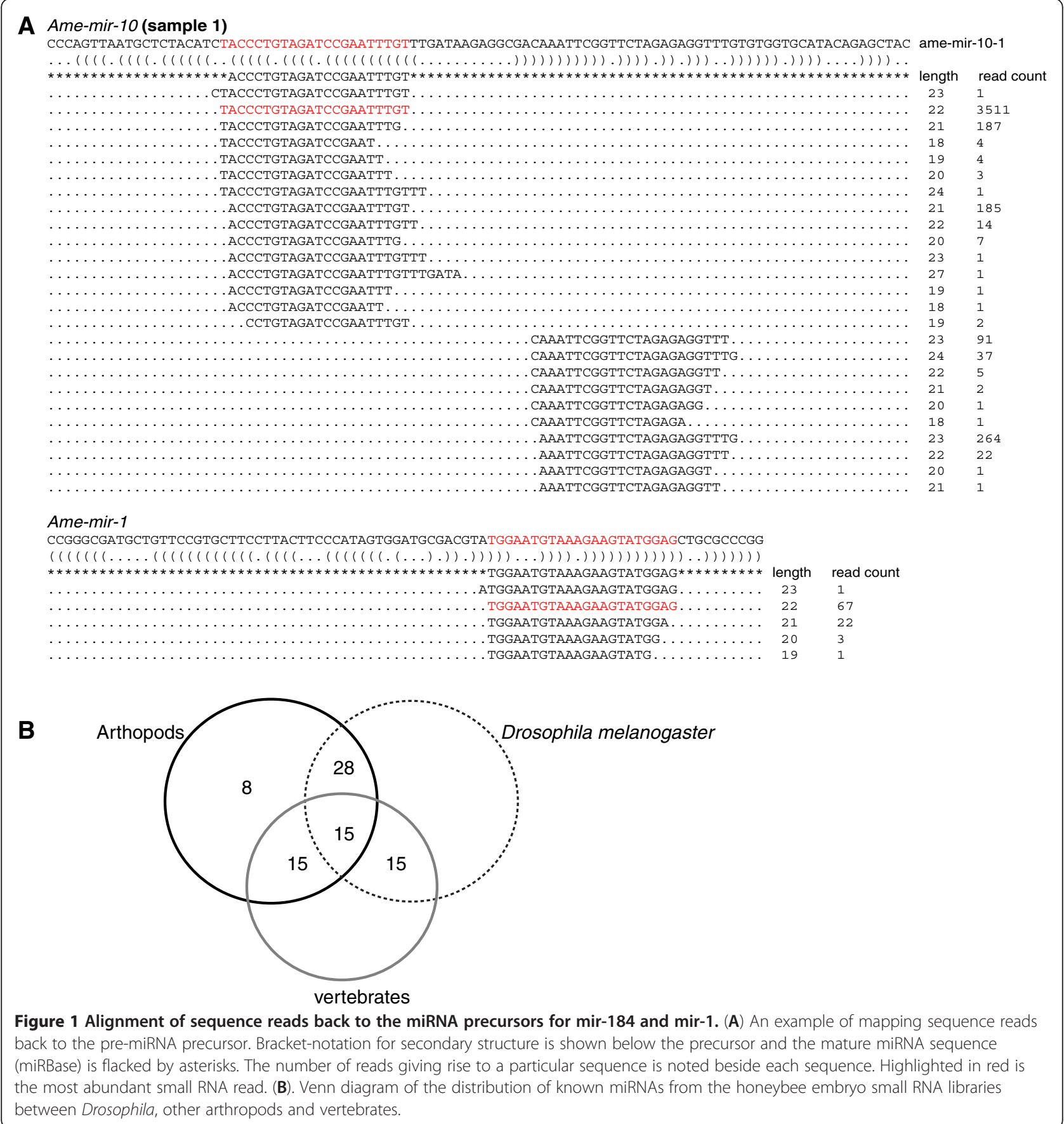

miRNAs have so far been predicted. The Drosophila genome encodes at least 430 miRNAs (mirBase). We examined the expression of honeybee miRNAs during early development, by deep sequencing and in situ hybridization. This included developmental stages at which the anterior-posterior and dorso-ventral axes have been established, patterning including segmentation is underway, just prior to gastrulation. As the pattern of miRNA expression is often reflective of their function in a developmental process or tissue patterning, we examined the expression of eight miRNAs identified in our study. Additionally, RNAi knockdown of Dicer during early embryogenesis indicated that small RNAs are likely to contribute to early honeybee embryo development.

\section{Results and discussion}

Abundance and expression of previously known miRNAs Total RNA was extracted from honeybee embryos aged from 24 to 30 hours old (stages 4 to 5). Approximately 


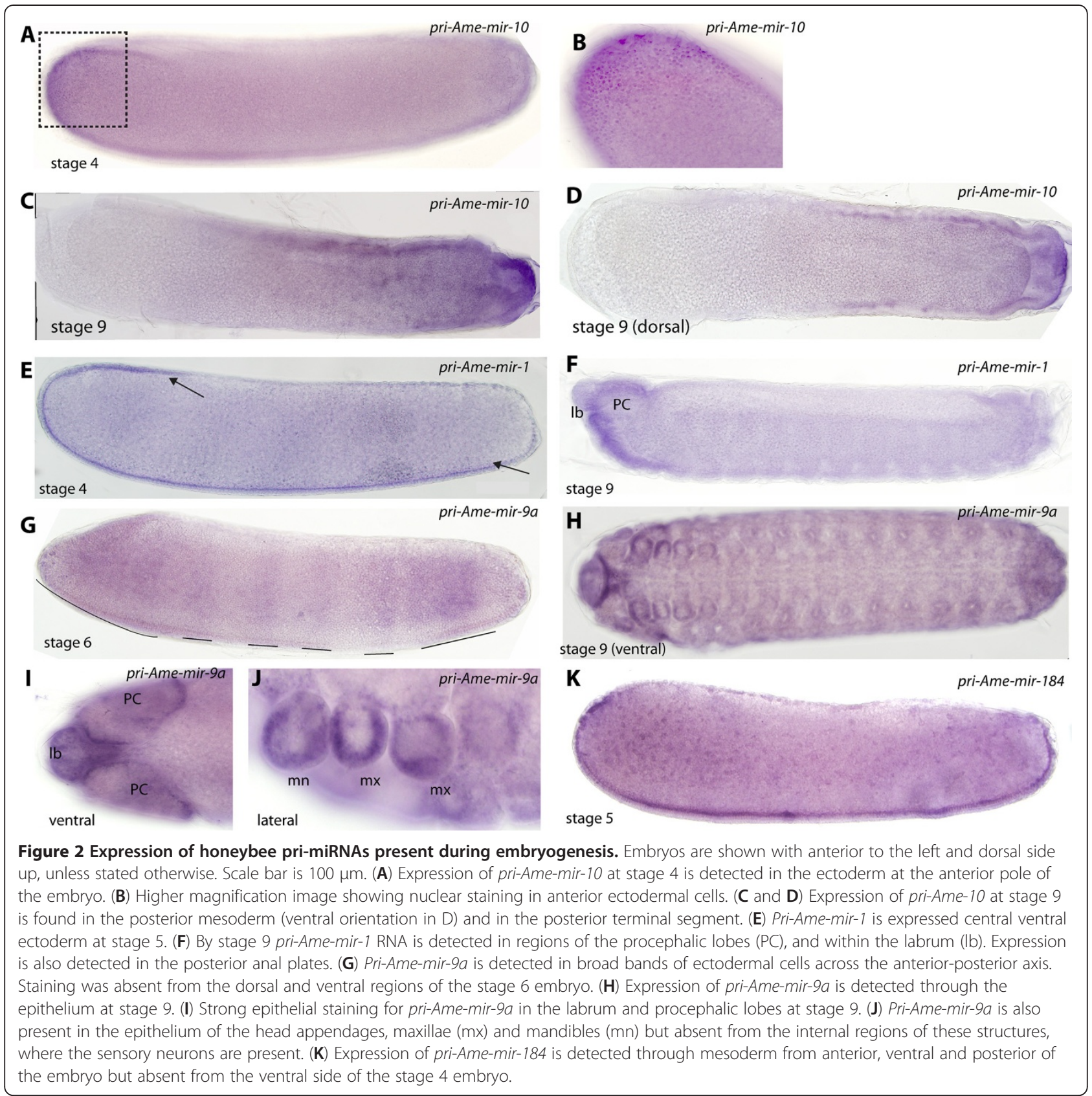

20 million reads were generated per sample, corresponding to 3 million unique reads (Table 1 ). Almost $70 \%$ of unique small RNA tags were mapped back to the Apis mellifera genome (Table 1). The number of reads for known (i.e. present in miRBase) Apis mellifera miRNAs are shown in Table 2 and an example of an alignment is shown in Figure 1A. This provides further experimental evidence that these are transcribed miRNAs in the honeybee genome. Of the 168 mature miRNA in miRBase, 45 were represented in both samples. This indicates that almost $28 \%$ of known miRNAs are expressed in the early honeybee embryo, implying a possible role for these in regulating developmental pathways. An example of read alignments to precursor miRNA are shown Figure 1A.

We determined if these miRNAs are also present in the genomes of other phyla. Of the miRNAs we isolated from honeybee embryos, 36 are also present in the genomes of other arthropods. 15 of these are present in the vertebrate lineage, indicating these are likely to be ancestral (Figure 1B). Less conservation was observed between Apis and Drosophila miRNA content which has also been noted previously between Tribolium and Drosophila [18], indicating that Arthropod groups 
Table 3 Novel miRNA profile and presence $(+)$ or absence $(-)$ in the genomes of other insects

\begin{tabular}{|c|c|c|c|c|c|}
\hline Candidate & Reads & Reads & Bombus & Nasonia & Atta \\
\hline miRNA & $\overline{\text { Sample } 1}$ & $\overline{\text { Sample } 2}$ & & & \\
\hline mir-0002 & 197 & 144 & + & - & - \\
\hline mir-0003 & 106 & 82 & + & - & - \\
\hline mir-0004 & 7 & 8 & + & - & - \\
\hline mir-0005 & 1642 & 2263 & + & + & + \\
\hline mir-0006 & 30 & 35 & - & - & - \\
\hline mir-0007 & 5688 & 5036 & + & - & - \\
\hline mir-0008 & 59 & 29 & + & - & + \\
\hline mir-0009 & 6 & 9 & + & - & - \\
\hline mir-0010 & 44 & 30 & - & - & - \\
\hline mir-0011 & 6 & 14 & - & - & - \\
\hline mir-0012 & 2095 & 1152 & - & - & - \\
\hline mir-0013 & 10 & 17 & - & - & - \\
\hline mir-0014 & 405 & 206 & - & - & - \\
\hline mir-0017 & 405 & 206 & - & - & - \\
\hline mir-0018 & 37 & 10 & - & - & - \\
\hline mir-0020 & 87 & 88 & + & - & - \\
\hline
\end{tabular}

(other than Diptera) have more miRNA content in common to each other than when compared separately to Diptera.

To determine if the developmental expression patterns of miRNAs with orthologues in both honeybee and Drosophila were conserved, we examined the expression of four miRNAs detected in our study, with expression and functional data of their orthologue in Drosophila.

In situ hybridisation was performed using RNA probes designed to bind to the pri-miRNA, the longer transcript from which the pre-miRNA is produced, prior to export from the nucleus. This strategy has been successfully used in previous studies illustrating that it reflects the mature miRNA expression (when detected using LNA probes) [25]. The probes used detect nascent transcripts before processing by Drosha RNase III enzyme and thus are expected to detect nuclear dots rather than the cytoplasmic staining produced with probes against an mRNA transcript (for example see Additional file 1: Figure S3).

Mir-10 is a widely conserved miRNA in both sequence and genomic location in both invertebrates and vertebrate Hox gene clusters. It is located within the Hox complex in Drosophila between the deformed ( $\mathrm{dfd}$ ) and sex-combs reduced (Scr) Hox genes and has been predicted to directly regulate mRNA translation of nearby Hox genes that contain mir-10 binding sites in their 3'UTRs [26]. However ectopic expression of Dme-mir-10 had no significant effect on the expression of predicted Hox targets (Scr and Abd-B) [25], suggesting that they may not be biologically relevant targets for mir-10 in a laboratory setting. Apis mellifera mir-10 (Ame-mir-10) is also located in the Hox gene complex between $A m$-dfd and Am-Scr (Additional file 2: Figure S4). We detected expression of this miRNA during embryogenesis (Table 1 and Figure 1A). In situ hybridisation analysis revealed expression of pri-Ame-mir-10 at the anterior pole of the embryo (Figure 2A and B). Later pri-Ame-mir-10 RNA expression (stage 9; approximately 48 hours old) was detected throughout the posterior two-thirds of the embryo, in the posterior terminal segment and the underlying mesoderm (Figure 2D). This is consistent with expression of a Hox regulator, as it shows limited expression along the anterior-posterior axis. In comparison, Drosophila pri-Dme-mir-10 expressed in a broad band across the middle of blastoderm embryos and, following germ band extension, it is expressed in the posterior half of the embryo, in the anal pad, ventral neuroectoderm and hindgut [25]. This indicates that while there are similarities in late embryonic expression of mir-10 between Drosophila and honeybee embryos, mir-10 expression in early embryos is quite different. This may indicate a shift in mir-10 function in early embryogenesis between Diptera and hymenoptera.

Previous deep sequencing of Drosophila embryonic RNA revealed that most of the reads correspond to the $3^{\prime}$ arm of the mir-10 precursor [27], although RNA expression patterns of both mir-10-5p and mir-10-3p is similar in Drosophila embryos [25]. We found that the $90 \%$ of sequence reads are from the $5^{\prime}$ arm of the premiRNA in honeybee embryos, indicating that mir-10-5p is responsible for the majority of mir-10 function (Figure 1). This has also been found for mir-10 in Tribolium [18]. Changes to which part of the pre-miRNA strand provides the dominant or functional miRNA sequence (arm switching) is proposed to be one mechanism of miRNA evolution to drive miRNA diversification [28]. Our results and those from Tribolium [18] would indicate that the ancestral dominant arm was the mir10-5p (producing the mature miRNA) and that this has switched during Drosophila evolution to the mir-10-3p arm. However, while only $10 \%$ of the total reads for pre-mir-10 were from the $3 \mathrm{p}$ arm in honeybee embryos (Figure 1), it was still a significant number (425) and more abundant that some of the other miRNAs detected (Table 2), indicating that the mature miRNA from this arm of the mir-10 hairpin (mir-10-3p) may have a distinct role during honeybee development.

Mir-1 is a highly conserved miRNA that has been suggested to play a role in myogenesis in Drosophila and vertebrates [29-31]. We detected low numbers of reads for Ame-mir-1 in both our samples (Table 1), but because of its conservation between vertebrates and invertebrates, we 
examined its RNA distribution. Staining for pri-Ame-mir-1 was weakly detected through ventral and anterior mesoderm of the embryo (Figure 2E). By stage 9, expression was found in the anterior of the embryo within the area of the labrum and in restricted regions of the procephalic lobes (Figure 2F). No expression was detected in mesodermderived tissues late in development. In Drosophila, Dmemir-1 is also expressed in ventral mesoderm [29,32] but continues to be expressed throughout mesodermal tissues later in development and is required for muscle and cardiac patterning $[29,33]$. This expression pattern of Dmemir-1 is regulated by Twist (Twi), a pro-mesoderm transcription factor [29]. Examination of the upstream and downstream regions surrounding the Ame-mir-1 coding region failed to find any significant cluster of Twi binding sites (Additional file 3: Figure S5), possibly explaining the lack of Ame-mir-1 expression in the Apis mesoderm. This implies loss of an enhancer element(s) for directed expression in ventral mesoderm in the honeybee, as mesodermal expression is also found in vertebrates suggesting it is a more ancient pattern for mir-1.

Mir-9a is a conserved microRNA in sequence but with differing functions in invertebrates and vertebrates. Drosophila Dme-mir-9a is expressed in the dorsal ectoderm and neuro-ectoderm at early stages [32] and ectodermal epithelial cells including the epithelial surfaces of the head appendages. Dme-mir-9a homozygous mutants survive to hatching and are fertile but produce ectopic sensory neurons, indicating a role in negatively regulating neuron number [34]. In vertebrates, however, while mir$9 \mathrm{a}$ is expressed in the developing brain it has a differing role, positively regulating neurogenesis $[35,36]$. Sequencing data indicated that Ame-mir-9a was expressed (Table 1) during honeybee early development. In situ hybridisation at stage 5 , just prior to gastrulation, detected mir-9a throughout the head ectoderm, and then in broad ectodermal stripes across the middle of the embryo to the posterior terminus (Figure 2G). Expression was

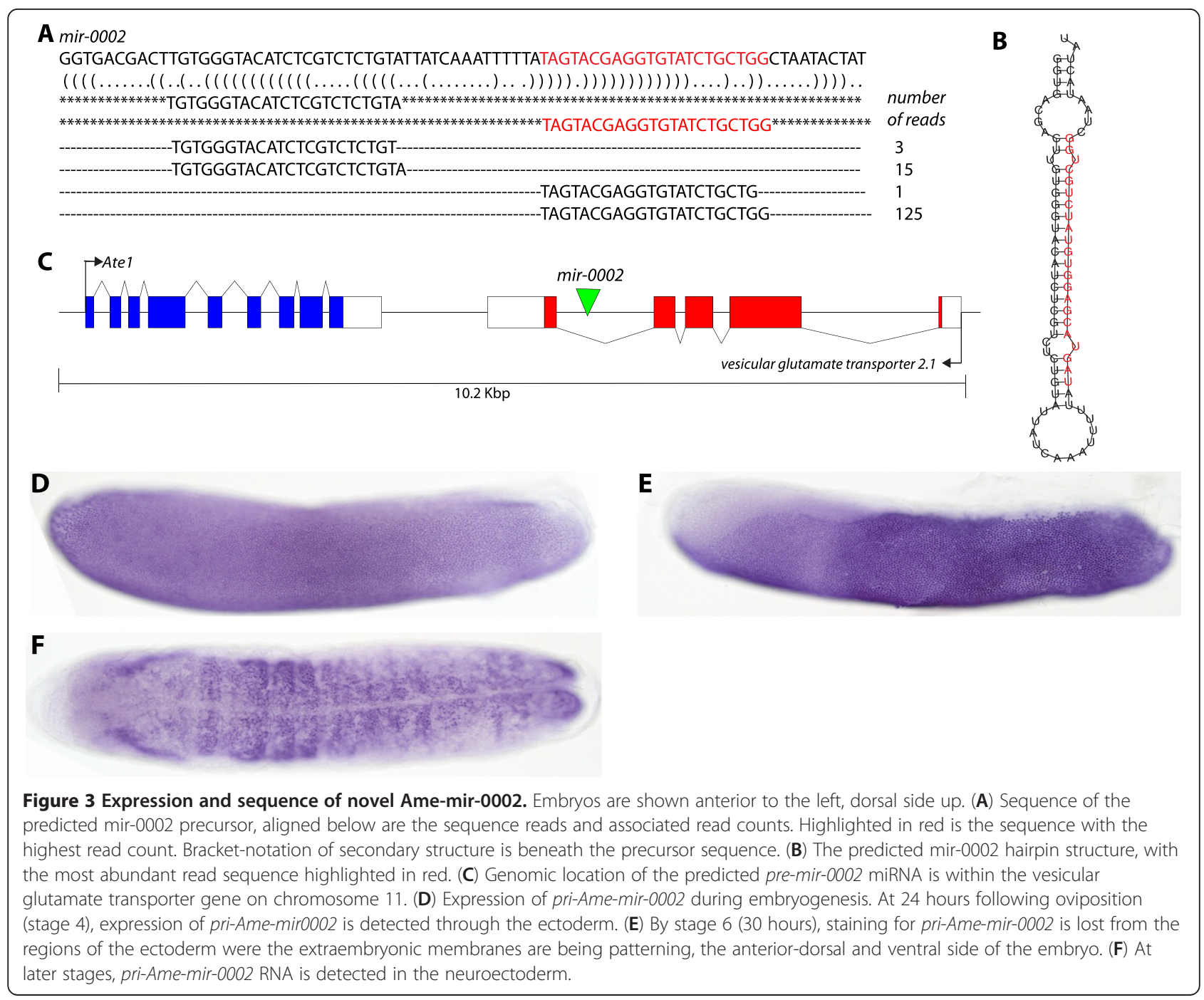




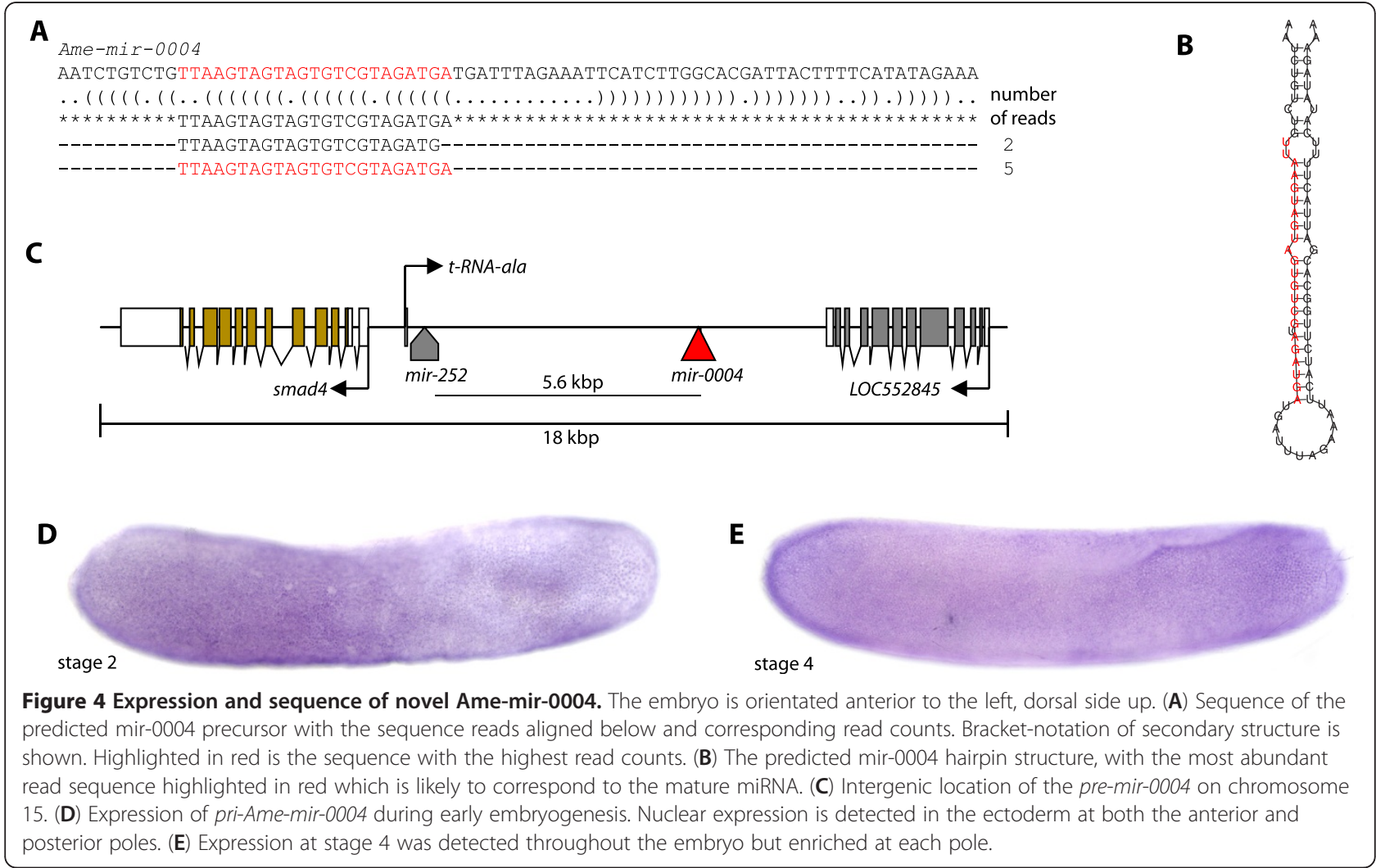

absent in the dorsal and ventral sides of the embryo (Figure 2G). By stage 9, pri-Ame-mir-9a RNA was found throughout the epidermis but was weak or absent in neurons of the central nervous system (CNS) (Figure 2H). Expression was strongest in the epidermis of the procephalic lobes and labrum, and no staining was found within cephalic and labrum regions where the neuronal cells are present (Figure 2I). A similar pattern of expression was also found in the mandibles and maxillae, with all appendages exhibiting strong staining around epithelium for pri-Ame-mir-9a but absent from the central regions of the appendages, where the sensory neuronal tissue are predicted to be (Figure 2J). Therefore mir-9a RNA expression in both honeybee and Drosophila show similar patterns, with strong epithelial cell basis, consistent with a conserved role in regulating production of sensory organ neuronal cells and suppressing sensory neural fate in the surrounding epithelia. In vertebrates, mir-9a has a quite different role in positively regulating neurogenesis, indicating that both its expression and function has changed significantly in the vertebrate group or this developmental role of mir-9a is particular to the insect group.

Mir-184 is also conserved between invertebrates and vertebrates, and has been shown to play an important role in axis formation and oogenesis in Drosophila [37]. Ame-mir-184 had the highest read count of any miRNA in our small RNA library (Table 1). Pri-Ame-mir-184 RNA was detected in the mesodermal cells throughout the embryo except the dorsal side of the embryo where extraembryonic membranes differentiate (Figure $2 \mathrm{~K}$ ). Previous studies have shown that Dme-mir-184 is expressed along the mesoderm on the ventral side of the embryo $[32,37]$.

\section{Prediction and expression of novel miRNAs}

Candidate miRNAs were identified in our small RNA libraries using the prediction software Mireap [38] that takes into consideration typical miRNA features, such as the presence of a Dicer cleavage site, and correct secondary structure. Sixteen previously unknown miRNAs satisfied these criteria, were present in both RNA samples (Table 3; Additional file 4), and were mapped back to the genome. To determine if these miRNAs were also present in other insects, we used BLAST searches with the mature microRNA sequence to hymenopteran genomes, and then examined the presence of a stem-loop structure in the predicted precursor for each significant hit to determine if they are likely to represent orthologous miRNAs. Eight novel AmemiRNAs were also present in the Bombus (Bumble bee) genome and two within Atta cephalotes (Ant) genome but only one within the Nasonia (Jewel wasp) genome (Table 3). This indicates that many of these novel miRNAs may have arose after branching of the Apoidea 
group or have been lost from other lineages. We examined the expression patterns of four of these newly identified miRNAs during honeybee embryogenesis.

The novel miRNA Ame-mir-0002 is located within an intron of the vesicular glutamate transporter 2.1 gene on chromosome 11 (Figure 3C). Pri-Ame-mir-0002 RNA was expressed throughout the ectoderm of the stage 4 embryo (Figure 3D), by late stage 5 RNA expression is no longer detected at the anterior-dorsal region of the embryo, or along the dorsal surface (Figure 3E), this includes areas where the extraembryonic membranes form [22]. Later pri-Ame-mir-0002 RNA expression was found throughout the neuroectoderm (Figure 3F). These expression patterns imply a possible role for mir-0002 in patterning the ectoderm and neuroectoderm during honeybee development. Many species-specific miRNAs are located in the introns of protein coding genes compared to those are proposed to be more ancient which tend to be intergenic [39]. Most are often transcribed from the host-gene promoter, although some intronic miRNAs have their own promoter [40,41]. Further work is required to determine if mir-0002 expression is driven from the host-gene promoter or if it has its own promoter region.

The novel miRNA mir-0004 is on chromosome 15, located between smad4 and a hypothetical gene, and about $5.6 \mathrm{kbp}$ from mir-252 (Figure 4C). Expression of pri-Amemir0004 RNA was detected in ectoderm cell nuclei at the anterior and posterior half of the embryo, but weaker within the central region of the embryo (Figure 4D). Embryo staining produced high background, probably as a result of longer staining times required to visualize the RNA expression; read count from the small RNA libraries indicated mir-0004 was present at low levels.

micr-0007 is located in the intergenic space between two predicted genes (Figure 5A). Pri-mir-0007 RNA was detected in the dorsal-posterior ectoderm (Figure 5D), a part of the embryo where the amnion arises [22]. By stage 9, pri-mir-0007 RNA was detected in the posterior half of the amnion (Figure 5E).

Mir-0005 was detected with high numbers of reads (1642 and 2263 reads per sample) in our small RNA

A Ame-mir-0007 AGCGTGCCTCTTTCGAGGCTGTTTAAATGGATTCACATTGTGCAACTCGACGGCATAAATCCATTTAAGTCGTTCGAAAGAGGCTGGCTGT

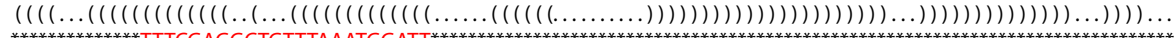

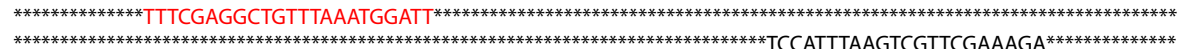
--TTTCGAGGCTGTTTAAATGGA---TTTCGAGGCTGTTTAAATGGAT-

--o---1TTCGAGGCTGTTTAAATGGATT--

--TTTCGAGGCTGTTTAAATGGATTCA-

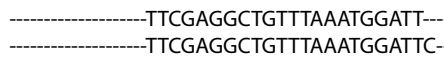

--TTCGAGGCTGTTTAAATGGATTCA-

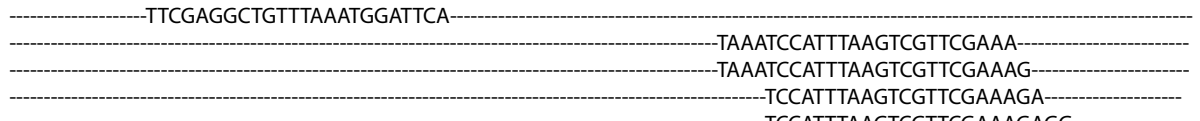

ATCGTTCGAAAGA

--TCCATTTAAGTCGTTCGAAAGAGG

number

of reads

18

24

8

101

3833

33

514

88

15

275

7

3
74

74

C
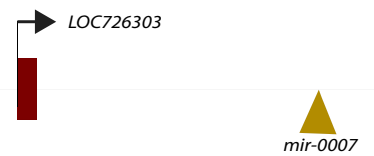

LOC410888

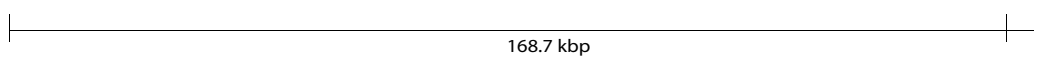

D

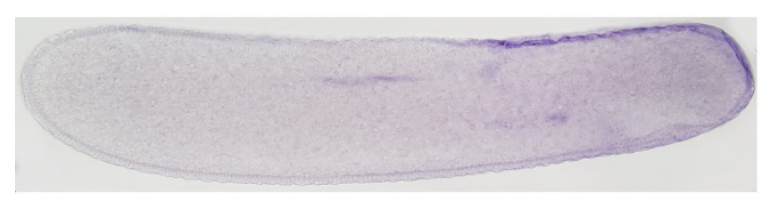

E

Figure 5 Expression and sequence of novel Ame-mir-0007. Embryos are positioned anterior to the left, dorsal side up. (A) Sequence of the mir-0007 precursor with aligned sequence reads and read counts. Highlighted in red is the sequence with the highest read counts. Bracketnotation of secondary structure is shown below the precursor. Mature miRNA and mirRNA* sequences are shown surrounded by asterisks. (B) The predicted mir-0007 hairpin structure, the most abundant read sequence highlighted in red. (C) Genomic location of the pre-mir-0007; mir-0007 is located between the predicted genes LOC726303 and LOC410888 on chromosome 2. (D) Expression during embryogenesis at stage 4. (E) Expression of pri-mir0007 at stage 9 is though the posterior of the extraembryonic membrane. 
libraries. Analysis of the genomic location and mir-0005 mature sequence indicates that this is likely to be a paralogue of mir-92b (Figure 6A). Previously two mir-92b-1 genes had been identified in the honeybee genome (mirBASE) but these are located on different chromosomes to that of mir-92a (Additional file 5: Figure S7), whereas mir-0005 is located next to mir-92a and had not been reported previously. We examined the conservation of the mir-92a -92b cluster in arthropods (Figure 7). In aphid, Apis, Tribolium, Nasonia and Bombus, mir-92a and mir-92b are clustered within $200 \mathrm{bp}$ of each other in an intron. In Diptera (Drosophila, Aedes and Anopholes), they are no longer linked and are now separated by 5 to $50 \mathrm{kbp}$ of DNA. This is similar to previously studies that indicate that miRNA clusters are conserved in most insects but become fragmented in Diptera lineage [18]. We found that Mir-92a was present at an almost tenfold lower read count in our small RNA libraries compared to mir-0005/mir092b, indicating they are produced differentially despite being close enough to be processed from the same primary transcript (Figure 7 and Additional file 5: Figure S7). The pri-mir-0005 probe used to detect expression of RNA would detect a primary transcript that includes both mir-92a and mir0005. Nuclear staining for pri-mir-0005 RNA in honeybee was found to be present at the posterior ectoderm of stage 4 embryos (Figure $6 \mathrm{C}$ and D). Later, at stage 9 , pri-mir-0005 RNA expression was detected in the amnion (Figure 6F).

\section{Knockdown of Dicer during early embryogenesis}

To determine if miRNAs expressed during embryogenesis do indeed play a role in honeybee development, we knocked down dicer expression using siRNAs against Am-dicer (Figure 7A). Dicer is a ribonuclease required for the production of mature miRNAs from the pre-miRNA precursor, thus by reducing levels of Dicer in the early embryo, we expect to target the processing of pre-miRNA precursors and thus decrease miRNA synthesis. Freshly laid honeybee embryos (approximately 400 each) were injected with siRNAs against Am-dicer, or a control siRNA (Figure 8; Additional file 6). This strategy is likely to have resulted in knockdown of the zygotic production of small RNAs. Following injection, embryos were incubated for 48 hours and fixed and staining with DAPI

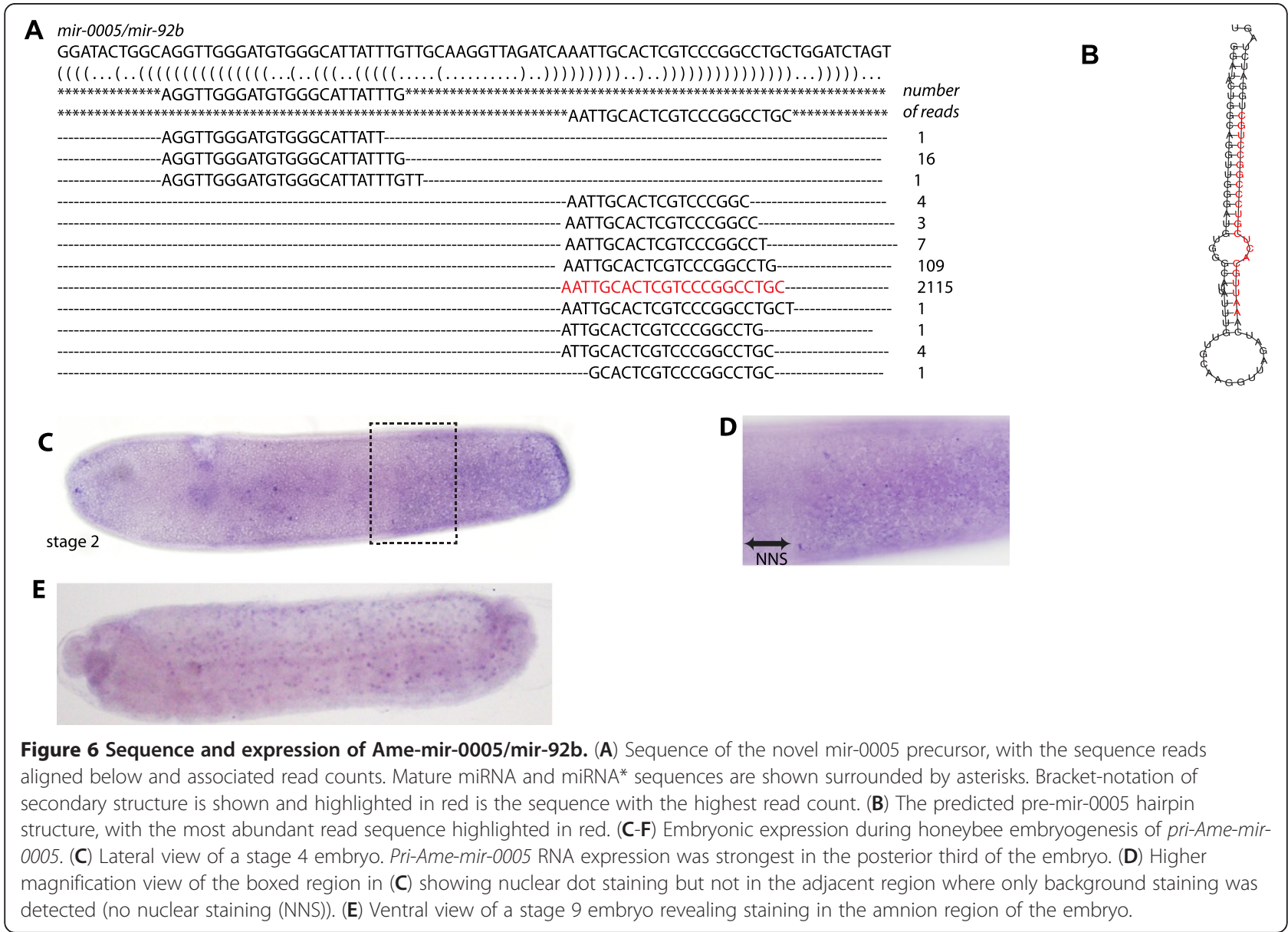




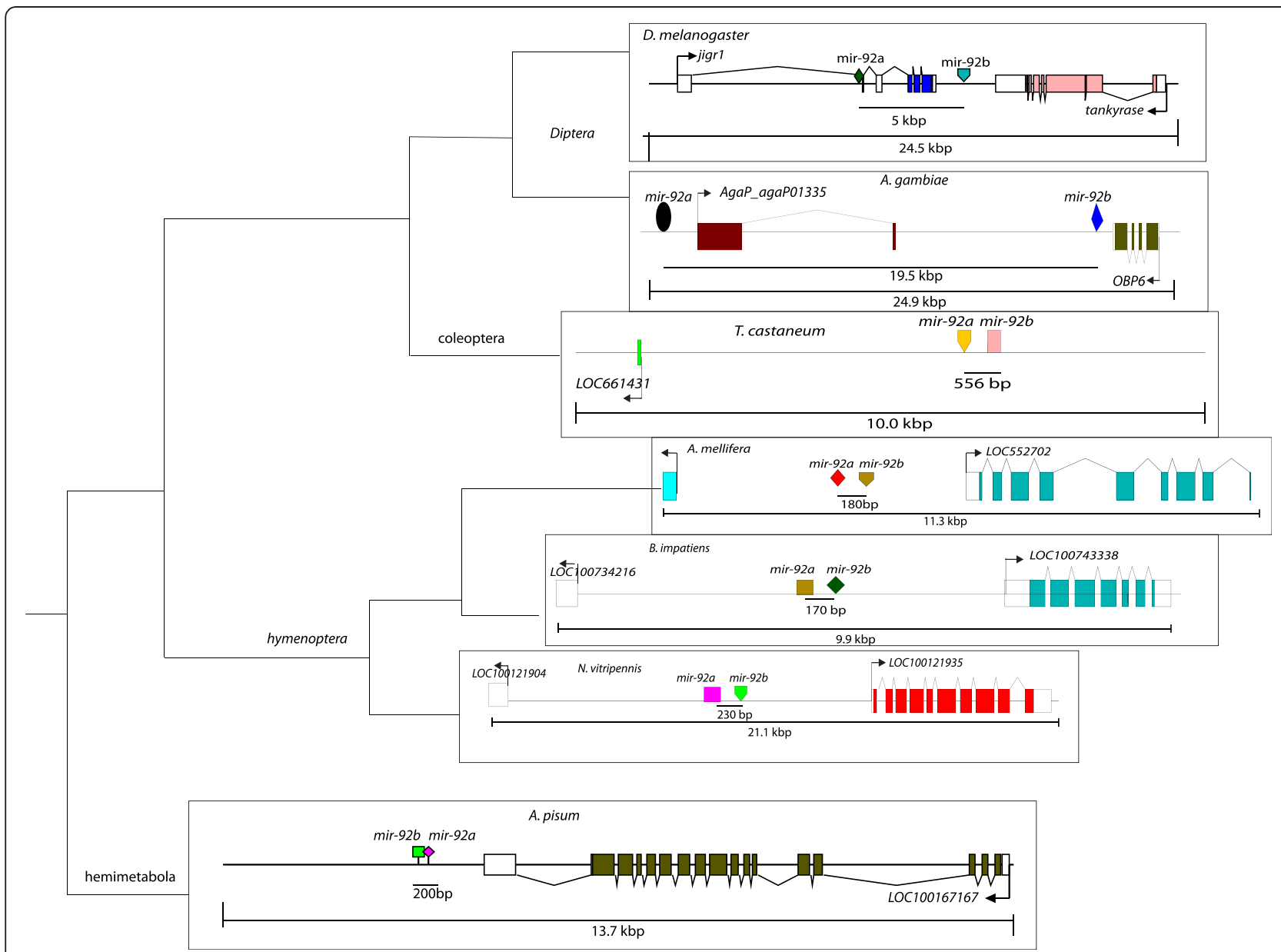

Figure 7 Genomic location of pre-mir-92a and pre-mir-92b in arthropods. Mir-92a and $b$ are intragenic positioned closely in hemimetabola, hymenoptera and coleopteran genomes and are predicted be expressed as part of the same pri-RNA transcript. However in Diptera, mir92a and $b$ are now separated by considerable distances and may no longer be part of the same polycistronic transcript.

to assess morphology. Approximately 20\% survived through to stage 9 , in line with previous survival ratios from similar procedures due to the nature of the injection procedure [22,23]. Posterior and anterior regions of the embryos were malformed with loss of terminal patterning (Figure $8 \mathrm{~B}, \mathrm{C}, \mathrm{F}$ and $\mathrm{H}$ ). In some embryos there was a significant reduction in the amnion that normally covers the yolk sac, and the dorsal regions of the embryo extend further towards the ventral side of the embryo (Figure $8 \mathrm{~B}$ and $\mathrm{H}$ ). This phenotype is very similar to that of Am-zen RNAi knockdown [22], suggesting problems with dorso-ventral patterning. Abdominal tracheal pits were present but segments appeared to be fused together, indicating a defect in anterior-posterior patterning (Figure $8 \mathrm{~B}$ and $\mathrm{C}$ ).

\section{Conclusions}

We examined the miRNA content of early honeybee embryos by deep sequencing followed by determination of the expression patterns of eight of these miRNAs.
Consistent with both miRNA expression patterns and target prediction, Dicer siRNA knockdown embryos had defects in extraembryonic membrane formation, anterior-posterior and dorso-ventral patterning, suggesting that miRNAs may have functions in regulating these patterning pathways or their gene target(s) have roles in multiple pathways.

Many miRNAs that are expressed during embryo development are deeply conserved throughout metazoans indicating a more ancient origin. However, several are unique to the arthropod group, and some restricted just to the Apis lineage. Given the developmental expression of these miRNAs, they may have taken on roles particular to the development of the honeybee embryo. Previous studies have hypothesised that miRNAs are continuously added to the metazoan genomes, are stabilized once added, and are rarely lost $[27,42,43]$.

Interestingly, some of the highly conserved miRNAs identified in our study had very different expression 


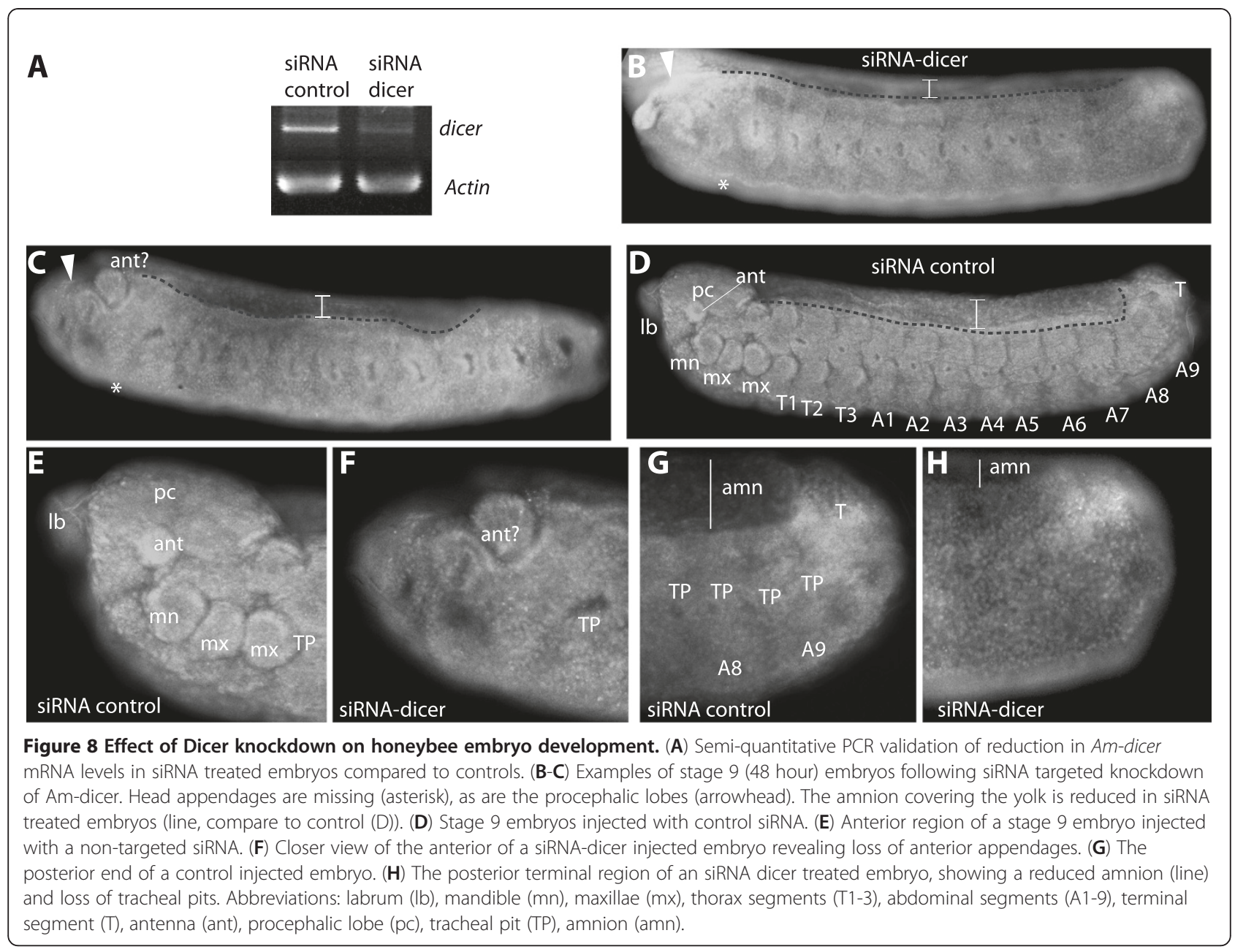

patterns in the honeybee to those documented in other animals. Mir-1 expression differed between Apis and other animals, which may result from loss of a regulatory element or binding sites for cis-regulatory proteins [44]. Early embryonic expression of mir-10 differed between Drosophila and Apis, but later expression was similar and is consistent with regulation by separate cisregulatory elements (one controlling early expression, one controlling later expression) as suggested previously for Drosophila mir-10 [8]. This suggests that earlier expression regulatory elements are evolving more rapidly. A similar pattern of more labile expression in early development has previously observed for protein-coding genes [24,45-47]. These changes or shifts in miRNA expression imply the regulatory regions controlling miRNA expression are also rapidly evolving. This indicates the importance that changes to gene-regulatory sequences contribute to the evolution of developmental pathways extends to also changes associated with regulatory elements that control miRNA genes.

\section{Methods}

\section{Sample collection and preparation}

A queen honeybee was caged with an empty area of an Eziqueen queen rearing frame and placed back into the hive. After 5 hours the frame was removed and the queen released back into the hive. The eggs were removed and while still attached to the black strips of the Eziqueen frame incubated for 24 hours. Eggs were collected and total RNA extracted using TRIzol (Life Technologies). Total RNA concentration and purity determined was using a Nanodrop spectrometer (Thermo Scientific). $10 \mu \mathrm{g}$ of purified total RNA was sent to Beijing Genomics Institute (BGI) for sequencing on an Illumina HiSeq 2000 sequencer. Low quality reads, reads without the adaptors, reads with polyA sequences and reads without the insert tag where removed. Also discarded were any tRNA, rRNA, snRNA and snoRNA sequences. Sequence reads were mapped to the genome using the programme SOAP [44]. Small RNA tags were aligned to known miRNA Apis mellifera precursors 
(miRBASE). The two small RNA libraries shared 98.06\% of sequences. Analyses of the length distribution of cleaned reads showed enrichment of small RNAs from 22 to 31 nucleotides (Additional file 7: Figure S1); we would expect about a length of 22nt for miRNAs. Examination of the first base of the 22 nucleotide sequences revealed most show a first base bias to uridine as predicted from previous deep sequencing miRNA studies (Additional file 8: Figure S2).

\section{Amplication of pri-miRNA fragments}

Oligonucleotide primers were designed to amplify 500800 bp regions using genomic template. In each amplicon, the precursor miRNA resides in the centre of the sequence. PCR fragments were cloned into the vector pBluescript II KS (+/-) and sequenced. Cloned fragments were used to produce RNA sense and antisense in situ hybridisation probes. Oligonucleotide primer pairs were as follows: mir-10 5'ACAAATGGACGACGAAGAGG3' and 5'GCGGCACGTACGTTACTTTA3 ${ }^{\prime}$, mir-1 5'GCC ACGTACGTTCGAAAACT3 ${ }^{\prime}$ and 5'TTCGCAAGACG GATACATCA3', mir-184 $5^{\prime}$ GCCTCGGGTTTCGAGG CGTT3' $^{\prime}$ and $5^{\prime}$ AGGAGAAGGGAAGAATGTGCAGA GA3', mir-9a 5'CCGATTTCTCCGTCTTTTCTG3' and 5'CCGATTTCTCCGTCTTTTCTG3', mir-0002 TGTA CGGGCAGTACTGGG and TCTTGATGATGCGTCT TG, mir-0004 5'CAACGATGCGTTTCGACTTA3' and 5'GTACCCACGAGTCGTCAC3', mir-0005 5'TCGATA TTCGAAACGCAACA $3^{\prime}$ and $5^{\prime}$ TGG A TT TGAAT TC GTGTATGAAA3 ${ }^{\prime}$, mir-0007 5'ACGAGGATACACGG ATGGAC3 ${ }^{\prime}$ and CAAT TCAC TTCCTTTTCACCTCA3'.

\section{In situ hybridization on honeybee embryos}

Performed as per Osborne [48] with the following modifications. Incubations with pri-miRNA anti-sense and sense probes were carried out at $60^{\circ} \mathrm{C}$ with rotation for 48 hours before post-hybridisation wash steps to remove excess probe. Embryos were incubated overnight at $4^{\circ} \mathrm{C}$ with anti-dioxygenin-alkaline phosophatase antibody with rotation before post-antibody wash steps and colour reaction.

\section{Dicer siRNA knockdown}

Two siRNAs were designed against Am-dicer; GGAC GAAGAGUUAGAGUUAUU and UGAAACAGCUAG UGAUAUAUU. The two siRNAs were injected together at a final concentration of $5 \mu \mathrm{g} / \mathrm{ml}$ into freshly laid eggs attached to plastic strips from an Eziqueen frame [49]. As a control, a non-targeting siRNA (D001810-01-05, Dharmacon) was also injected at the same concentration. Following injection, embryos were placed in a humidified incubator at $35^{\circ} \mathrm{C}$ for 48 hours. After incubation they were fixed with heptane/formaldehyde in PBS overnight, rocking at room temperature.
After fixation, embryos were washed with PBS and stained with DAPI before visualization on an Olympus BX61 microscope with a DP71 camera. Embryos were staged as per DuPraw [19].

\section{Additional files}

\begin{abstract}
Additional file 1: Figure S3. (A) Pseudocoloured image showing DAPI (blue) and Am-eve RNA staining in red. Am-eve RNA is detected in the cytoplasm of embryonic cells. (B) Pseudocoloured image of pri-mir-0002 RNA staining (red) overlaid with DAPI (blue) staining. Pri-mir-0002 RNA is detected in the nucleus of embryonic cells.
\end{abstract}

Additional file 2: Figure S4. Genome location and read count for Ame-mir-10. Genome location of Ame-mir-10 in the Hox complex, between deformed (dfd) and sex-combed reduced $(S \mathrm{cr})$.

Additional file 3: Figure S5. Clusterdraw analysis of the upstream regions of Dme-mir-1 and Ame-mir-1. Cluster of twi binding sites using the clusterdraw programme [50] with background model either site at $D$. melanogaster (A) or A. mellifera (B). This programme has successfully identified cis-regulatory elements in Apis and Drosophila previously [22,50-52]. $P$ values cut off on the $Y$-axis and position in the sequence along the $x$-axis.

Additional file 4: Figure S6. Alignment of mir-0008 and mir-0005/ mir-92b pre-miRNAs. Abbreviations: Apis mellifera, Bombus impatiens, Atta cephalotes, Nasonia vitripennis, Drosophila melanogaster. Boxed are the mature miRNA sequences.

Additional file 5: Figure S7. Alignment of sequence reads to Apis mir-92a, mir-92b-1 and mir-0005/mir-92b pre-miRNAs.

Additional file 6: Table S1. Phenotype of surviving larvae (at 72 hours) following siRNA injections.

Additional file 7: Figure S1. Length distribution in both samples of clean small RNA reads.

Additional file 8: Figure S2. miRNA nucleotide bias at each position.

\section{Competing interests}

The authors declare they have no competing interests.

\section{Authors' contributions}

MJW performed most of the in situ hybridisations, siRNA experiments and designed the project and wrote the publication. LZ cloned and synthesized the in situ probes, performed some of the honeybee in situ hybridisations and was involved in the writing of the manuscript. PKD discussed the data and took part in writing the manuscript. All authors read and approved the final manuscript.

\section{Acknowledgments}

We would like to thank James Smith for critical reading of this manuscript. Otto Hyink for help with the honeybee hives. Cris Anderson for technical assistance. This work was supported by a University of Otago Research Grant to MJW.

Received: 7 August 2012 Accepted: 22 October 2012

Published: 2 November 2012

\section{References}

1. Birney $E$, et al: Identification and analysis of functional elements in $1 \%$ of the human genome by the ENCODE pilot project. Nature 2007, 447:799-816. doi:10.1038/nature05874.

2. Graveley BR, et al: The developmental transcriptome of Drosophila melanogaster. Nature 2011, 471:473-479. doi:10.1038/nature09715.

3. Jacquier A: The complex eukaryotic transcriptome: unexpected pervasive transcription and novel small RNAs. Nat Rev Genet 2009, 10:833-844. doi:10.1038/nrg2683.

4. Lee $Y$, Jeon K, Lee JT, Kim S, Kim VN: MicroRNA maturation: stepwise processing and subcellular localization. EMBO J 2002, 21:4663-4670. 
5. Lee YS, et al: Distinct roles for Drosophila Dicer-1 and Dicer-2 in the siRNA/miRNA silencing pathways. Cell 2004, 117:69-81.

6. Hutvagner G, Zamore PD: A microRNA in a multiple-turnover RNAi enzyme complex. Science 2002, 297:2056-2060. doi:10.1126/science.1073827.

7. Cannell IG, Kong YW, Bushell M: How do microRNAs regulate gene expression? Biochem Soc Trans 2008, 36:1224-1231. doi:10.1042/ BST0361224.

8. Aboobaker AA, Tomancak P, Patel N, Rubin GM, Lai EC: Drosophila microRNAs exhibit diverse spatial expression patterns during embryonic development. Proc Natl Acad Sci U S A 2005, 102:18017-18022. doi:0508823102 [pii] 10.1073/pnas.0508823102.

9. He X, He X, Yan YL, DeLaurier A, Postlethwait JH: Observation of miRNA gene expression in zebrafish embryos by in situ hybridization to microRNA primary transcripts. Zebrafish 2011, 8:1-8. doi:10.1089/zeb.2010.0680.

10. Lau NC, Lim LP, Weinstein EG, Bartel DP: An abundant class of tiny RNAs with probable regulatory roles in Caenorhabditis elegans. Science 2001, 294:858-862. doi:10.1126/science.1065062.

11. He $X$, et al: miR-196 regulates axial patterning and pectoral appendage initiation. Dev Biol 2011, 357:463-477. doi:10.1016/j.ydbio.2011.07.014

12. Chen $Z$, et al: DiGeorge syndrome critical region 8(DGCR8) -mediated miRNA biogenesis is essential for vascular smooth muscle cell development in mice. J Biol Chem 2012. doi:10.1074/jbc.M112.351791.

13. Zhao $Z$, et al: A negative regulatory loop between microRNA and Hox gene controls posterior identities in Caenorhabditis elegans. PLOS Genet 2010, 6. doi:10.1371/journal.pgen.1001089.

14. Shibata M, Nakao H, Kiyonari H, Abe T, Aizawa S: MicroRNA-9 regulates neurogenesis in mouse telencephalon by targeting multiple transcription factors. J Neurosci 2011, 31:3407-3422. doi:10.1523/ JNEUROSCI.5085-10.2011.

15. Shomron N: MicroRNAs and developmental robustness: a new layer is revealed. PLoS Biol 2010, 8:e1000397. doi:10.1371/journal.pbio.1000397.

16. Krek A, et al: Combinatorial microRNA target predictions. Nat Genet 2005 37:495-500. doi:10.1038/ng1536.

17. Heimberg AM, Sempere LF, Moy VN, Donoghue PC, Peterson KJ: MicroRNAs and the advent of vertebrate morphological complexity. Proc Natl Acad Sci U S A 2008, 105:2946-2950. doi:10.1073/pnas.0712259105.

18. Marco A, Hui JH, Ronshaugen M, Griffiths-Jones S: Functional shifts in insect microRNA evolution. Genome Biol Evol 2010, 2:686-696. doi:10.1093/ gbe/evq053.

19. DuPraw EJ: In Methods in developmental biology. Edited by Wilt FH, Wessells NK, Thomas Y. New York, USA: Crowell Company; 1967:183-218.

20. Osborne PW, Dearden PK: Expression of Pax group III genes in the honeybee (Apis mellifera). Dev Genes Evol 2005, 215:499-508.

21. Rushlow C, Levine M: Role of the zerknullt gene in dorsal-ventral pattern formation in Drosophila. Adv Genet 1990, 27:277-307.

22. Wilson MJ, Dearden PK: Diversity in insect axis formation: two orthodenticle genes and hunchback act in anterior patterning and influence dorsoventral organization in the honeybee (Apis mellifera). Development 2011, 138:3497-3507. doi:10.1242/dev.067926.

23. Wilson MJ, Dearden PK: Tailless patterning functions are conserved in the honeybee even in the absence of Torso signaling. Dev Biol 2009, 335:276-287. doi:S0012-1606(09)01174-9 [pii] 10.1016/j.ydbio.2009.09.002

24. Wilson MJ, Havler M, Dearden PK: Giant, Kruppel, and caudal act as gap genes with extensive roles in patterning the honeybee embryo. Dev Biol 2010, 339:200-211. doi:S0012-1606(09)01405-5 [pii] 10.1016/j. ydbio.2009.12.015.

25. Lemons D, Pare A, McGinnis W: Three Drosophila Hox complex microRNAs do not have major effects on expression of evolutionarily conserved Hox gene targets during embryogenesis. PLoS One 2012, 7:e31365. doi:10.1371/journal.pone.0031365.

26. Enright AJ, et al: MicroRNA targets in Drosophila. Genome Biol 2003, 5:R1. doi:10.1186/gb-2003-5-1-r1.

27. Ruby JG, et al: Evolution, biogenesis, expression, and target predictions of a substantially expanded set of Drosophila microRNAs. Genome Res 2007, 17:1850-1864. doi:10.1101/gr.6597907.

28. Griffiths-Jones S, Hui JH, Marco A, Ronshaugen M: MicroRNA evolution by arm switching. EMBO Rep 2011, 12:172-177. doi:10.1038/embor.2010.191.

29. Sokol NS, Ambros V: Mesodermally expressed Drosophila microRNA-1 is regulated by Twist and is required in muscles during larval growth Genes Dev 2005, 19:2343-2354. doi:gad.1356105 [pii] 10.1101/gad.1356105.
30. Tang $Y$, et al: MicroRNA-1 regulates cardiomyocyte apoptosis by targeting $\mathrm{BCl}-2$. Int Hear J 2009, 50:377-387.

31. Chen JF, et al: The role of microRNA-1 and microRNA-133 in skeletal muscle proliferation and differentiation. Nat Genet 2006, 38:228-233. doi:10.1038/ng1725.

32. Biemar F, et al: Comprehensive identification of Drosophila dorsal-ventral patterning genes using a whole-genome tiling array. Proc Natl Acad Sci U S A 2006, 103:12763-12768. doi:10.1073/pnas.0604484103.

33. Kwon C, Han Z, Olson EN, Srivastava D: MicroRNA1 influences cardiac differentiation in Drosophila and regulates Notch signaling. Proc Natl Acad Sci U S A 2005, 102:18986-18991. doi:10.1073/pnas.0509535102.

34. Li Y, Wang F, Lee JA, Gao FB: MicroRNA-9a ensures the precise specification of sensory organ precursors in Drosophila. Genes Dev 2006 20:2793-2805. doi:10.1101/gad.1466306.

35. Leucht $C$, et al: MicroRNA-9 directs late organizer activity of the midbrainhindbrain boundary. Nat Neurosci 2008, 11:641-648. doi:10.1038/nn.2115.

36. Deo M, Yu JY, Chung KH, Tippens M, Turner DL: Detection of mammalian microRNA expression by in situ hybridization with RNA oligonucleotides. Dev Dyn 2006, 235:2538-2548. doi:10.1002/dvdy.20847.

37. lovino N, Pane A, Gaul U: miR-184 has multiple roles in Drosophila female germline development. Dev Cell 2009, 17:123-133. doi:10.1016/j.devcel.2009.06.008.

38. Chen, et al: Identification and Characterization of novel amphioxus microRNAs by Solexa sequencing. Genome Biology 2009, 10:R78. doi:10.1186/gb-2009-10-7-r78. http://sourceforge.net/projects/mireap/.

39. Campo-Paysaa F, Semon M, Cameron RA, Peterson KJ, Schubert M: microRNA complements in deuterostomes: origin and evolution of microRNAs. Evol Dev 2011, 13:15-27. doi:10.1111/j.1525-142X.2010.00452.x.

40. Rodriguez A, Griffiths-Jones S, Ashurst JL, Bradley A: Identification of mammalian microRNA host genes and transcription units. Genome Res 2004, 14:1902-1910. doi:10.1101/gr.2722704.

41. Martinez NJ, et al: Genome-scale spatiotemporal analysis of Caenorhabditis elegans microRNA promoter activity. Genome Res 2008 18:2005-2015. doi:10.1101/gr.083055.108.

42. Sempere LF, Martinez P, Cole C, Baguna J, Peterson KJ: Phylogenetic distribution of microRNAs supports the basal position of acoel flatworms and the polyphyly of Platyhelminthes. Evol Dev 2007, 9:409-415. doi:10.1111/j.1525-142X.2007.00180x.

43. Wheeler BM, et al: The deep evolution of metazoan microRNAs. Evol Dev 2009, 11:50-68. doi:10.1111/j.1525-142X.2008.00302.x.

44. Li R, et al: SOAP2: an improved ultrafast tool for short read alignment. Bioinformatics 2009, 25:1966-1967. doi:doi:10.1093/bioinformatics/btp336. 1966-1967.

45. Dearden PK, et al: Patterns of conservation and change in honey bee developmental genes. Genome Res 2006, 16:1376-1384.

46. Wilkins A: The Evolution of Developmental Pathways. Sunderland, MA, USA: Sinauer Associates; 2002.

47. Davidson EH: The regulatory genome: gene regulatory networks in development and evolution. Burlington, MA, USA: Academic Press, Elsevier; 2006.

48. Osborne PW, Dearden PK: Non-radioactive in-situ hybridisation to honeybee embryos and ovaries. Apidologie 2005, 36:113-118.

49. Dearden PK, Duncan EJ, Wilson MJ: RNA interference (RNAi) in honeybee (Apis mellifera) embryos. Cold Spring Harb Protoc 2009, doi:2009/6/pdb. prot5228 [pii] 10.1101/pdb.prot5228. pdb prot5228.

50. Papatsenko D: ClusterDraw web server: a tool to identify and visualize clusters of binding motifs for transcription factors. Bioinformatics 2007 23:1032-1034. doi:10.1093/bioinformatics/btm047.

51. Papatsenko D, Goltsev Y, Levine M: Organization of developmental enhancers in the Drosophila embryo. Nucleic Acids Res 2009, 37:5665-5677. doi:10.1093/nar/gkp619.

52. Perry MW, Boettiger AN, Levine M: Multiple enhancers ensure precision of gap gene-expression patterns in the Drosophila embryo. Proc Natl Acad Sci U S A 2011, 108:13570-13575. doi:10.1073/pnas.1109873108.

doi:10.1186/1471-2148-12-211

Cite this article as: Zondag et al: Deep sequencing and expression of microRNAs from early honeybee (Apis mellifera) embryos reveals a role in regulating early embryonic patterning. BMC Evolutionary Biology 2012 $12: 211$ 